International Journal of Modern Physics E (C) World Scientific Publishing Company

\title{
The pionic Drell-Yan process: a brief survey
}

\author{
Wen-Chen Chang \\ Institute of Physics, Academia Sinica \\ Taipei 11529, Taiwan \\ changwc@phys.sinica.edu.tw \\ Dipangkar Dutta \\ Department of Physics and Astronomy, Mississippi State University, \\ Mississippi State, Mississippi 39762, USA \\ d.dutta@msstate.edu
}

\begin{abstract}
The Drell-Yan process is an invaluable tool for probing the structure of hadrons, and the pion-induced Drell-Yan process is unique in its sensitivity to several subtleties of the partonic structure. This is a brief review of the pionic Drell-Yan process with particular emphasis on the dilepton angular distributions and nuclear modification of the parton distributions (the EMC effect).

Keywords: pion; Drell-Yan; Lam-Tung relation; QCD vacuum; Boer-Mulders function; Sivers function; flavor dependence of EMC effect

PACS numbers: 12.38.Qk, 13.85.Qk, 13.88.+e, 14.20.Dh, 21.65.Cd, 24.85.+p, 25.80.Hp
\end{abstract}

\section{Introduction}

The observation of a continuum of massive lepton pairs in proton-uranium collisions ${ }^{1 / 2]}$ was explained by S. Drell and T.-M. Yan ${ }^{3[4}$ by the process that now bears their name. A leading order Drell-Yan parton model of collinear quark-antiquark $(q \bar{q})$ electromagnetic annihilation into a virtual photon successfully described the scaling behavior, the $A$-dependence of production cross section, and the polar angle distribution of leptons. Furthermore, the inclusion of the perturbative QCD effect of gluon emission and absorption by the partons could explain nicely, the transverse momentum distribution of lepton pairs and the $K$ factor (ratio of next-to-leading order to leading order cross section) enhancement of cross section above the naive Drell-Yan cross section. Hence Drell-Yan process has become one of the most intensively studied processes in $\mathrm{QCD}^{5-7}$ and an effective tool to study the partonic structure of hadrons, ranging from the Parton Distribution Functions (PDFs) ${ }^{8}$ to Transverse Momentum Dependent (TMD) distributions ${ }^{9}$ In the Drell-Yan process the partonic structure is probed via the time-like photon which is complementary to the space-like one in deep inelastic scattering. 
Moreover, since the production cross section of the Drell-Yan process scales as $\tau=m_{l \bar{l}} / s=x_{q} x_{\bar{q}}$, where $m_{l \bar{l}}$ is the dilepton invariant mass, $s$ is the square of the center-of-mass energy and $x_{q / \bar{q}}$ is the momentum fraction of the hadrons carried by the struck quark/anti-quark, and, since pions are composed of valance anti-quarks, the pion-induced Drell-Yan process is more effective in producing lepton pairs of large $m_{l \bar{l}}$, compared to the proton-induced process where the anti-quarks are sea quarks abundant only at relatively small $x$. In addition to yielding important information on the partonic structure of the target nucleons, pion-induced Drell-Yan process plays a unique role in exploring the pion structure, which is not accessible via deep inelastic scattering (DIS) where a stable target is required. The pioninduced Drell-Yan ${ }^{10}$ reaction has been studied at BNL, FNAL, CERN, and Dubna since 1979. In this article we review the results from five experiments: the CIP 1114 experiment at BNL, the NA3, ${ }^{[5 \mid 16}$ Omega $a^{17}$ and NA10?,18] 20] experiments at CERN and the E61521 experiment at FNAL. We will emphasize their results on the angular distributions of lepton pairs in Sec. 2, and the nuclear modification of quark distributions known as the EMC effect in Sec. 3,4 The experimental parameters of beam, target and range of dilepton invariant mass for these experiments are listed in Table. 1

Table 1. Experimental parameters of pion-induced experiments

\begin{tabular}{cccc}
\hline Experiment & Beam $($ Momentum $\mathrm{GeV} / c)$ & Target & $m_{l \bar{l}}\left(\mathrm{GeV} / c^{2}\right)$ \\
\hline CIP & $\pi^{ \pm}(80,225,253)$ & $\mathrm{C}, \mathrm{Cu}, \mathrm{W}$ & {$[3.0,9.0]$} \\
NA3 & $\pi^{ \pm}(150,200,280)$ & $\mathrm{H}, \mathrm{Pt}$ & {$[4.1,8.5]$} \\
NA10 & $\pi^{-}(140,194,286)$ & $\mathrm{W}, \mathrm{D}$ & {$[4.0,8.5]$} \\
Omega & $\pi^{ \pm}(39.5)$ & $\mathrm{W}$ & {$[2.0,2.7],[4.0,5.0]$} \\
E615 & $\pi^{-}(252)$ & $\mathrm{W}$ & {$[4.1,8.6]$} \\
\hline
\end{tabular}

Recent experimental and theoretical studies have enabled a multi-dimensional description of the partonic structure of nucleons: TMD distributions and Generalized Parton Distributions22 (GPD). In Sec. [5, we will discuss possible future measurements of the exclusive pion-induced Drell-Yan process and measurements using a transversely polarized target. Such measurements in parallel with DIS measurements can make unique and significant contribution to the construction of these next-generation parton distribution functions.

\section{Unpolarized Drell-Yan angular distribution}

Despite the success of perturbative QCD in describing the Drell-Yan cross sections, it still remains a challenge to fully comprehend the angular distributions of dilepton pairs. Assuming dominance of the single-photon process, the angular distribution of leptons from unpolarized Drell-Yan process could be denoted by the angular parameters $\lambda, \mu$ and $\nu$ as follows: 


$$
\frac{d \sigma}{d \Omega} \propto\left(1+\lambda \cos ^{2} \theta+\mu \sin 2 \theta \cos \phi+\frac{\nu}{2} \sin ^{2} \theta \cos 2 \phi\right)
$$

where $\theta$ and $\phi$ are the polar and azimuthal angles of the decayed leptons in the rest frame of virtual photon.

In the scheme of collinear $q \bar{q}$ electromagnetic annihilation, the produced virtual photon is purely transversely polarized and the angular distribution is proportional to $\left(1+\cos ^{2} \theta\right)$, i.e. $\lambda=1$ and $\mu=\nu=0$. The first measurement of polar angle distributions in the t-channel helicity frame or Gottfried-Jackson (GJ) frame by CIP experiment ${ }^{11}$ nicely confirmed the theoretical prediction and provided a strong support of Drell-Yan model in describing the dilepton production from the hadronhadron collisions.

Later it was also found by the $\mathrm{CIP} 12$ experiment that the polarization of virtual photon in GJ frame changed from transversely polarized $(\lambda=1)$ to longitudinally polarized $(\lambda=-1)$ when Bjorken- $x$ of anti-quark in the pion beam $\left(x_{\pi}\right)$ approached 1 . The magnitude of this variation was reduced in the Collins-Soper (CS) frame where the effect of intrinsic quark transverse momenta is minimized. This observation was not considered as conclusive in the subsequent measurements performed by the NA3 $\frac{15}{15}$ and the NA10 $\frac{18}{19}$ experiments. The controversy was finally settled by a clear observation of this phenomenon with good statistics in the E615 experiment ${ }^{21}$ as shown in Fig. 1.

In 1978, Lam and Tung studied the QCD-induced finite transverse momentum effect on the angular distributions of lepton pairs! 23 They found that QCD effect could lead to $\lambda \neq 1$ and $\mu, \nu \neq 0$ but the relation $1-\lambda=2 \mu$ in CS frame, so-called "Lam-Tung relation" holds for next-to-leading-order(NLO) QCD effect. Later studies showed that the Lam-Tung relation remains almost unchanged even up to NNLO! 26 Therefore this relation provides a unique opportunity to test the "QCD-improved quark-parton model", 23,25

The very first measurement by the NA3 experiment showed that $\nu$ increased strongly toward large transverse momentum of the lepton pair $\left(p_{T}\right)$ but the LamTung relation was roughly preserved. Nevertheless the following measurements with better statistics by the NA10 $\frac{18[19}{19}$ and the E615 $5^{21}$ experiments clearly identified a strong violation of Lam-Tung relation in the pion-induced Drell-Yan process with nuclei and deuterium targets. Fig. 2 shows E615 results of angular parameters $\lambda, \mu$ and $\nu$ as a function of $p_{T} / m_{l \bar{l}}$ and the degree of violation of Lam-Tung relation in the region of $0<p_{T}<5 \mathrm{GeV} / c, 4.05<m_{l \bar{l}}<8.55 \mathrm{GeV} / c^{2}$ and $0.2<x_{\pi}<1.0$. Clearly $\lambda$ deviates from 1 and both $\mu$ and $\nu$ have large non-zero values. The degree of violation increases with $p_{T}$. The fact that the violation was also observed using deuterium target $\frac{18}{19}$ excluded the possibility of a nuclear target effect. 

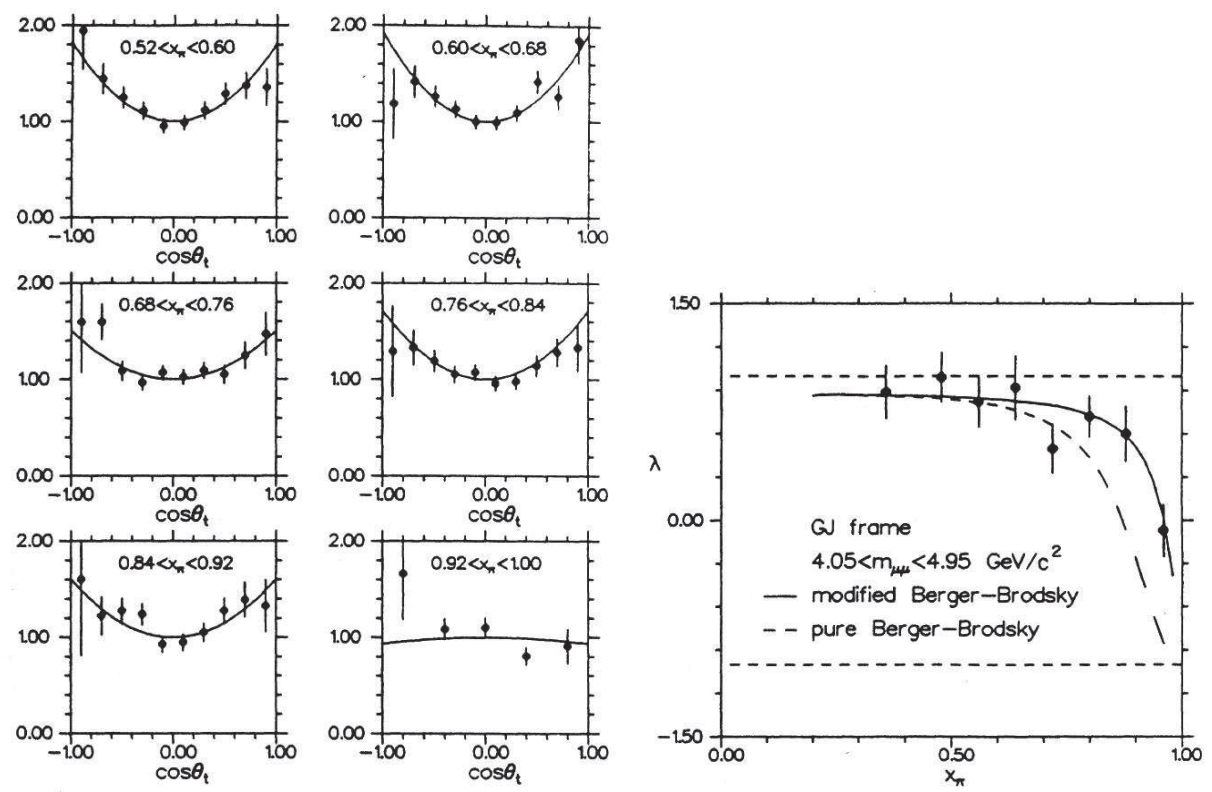

Fig. 1. (a) The GJ $\cos \theta$ distribution in regions of $x_{\pi}$ for $4.05<m_{l \bar{l}}<4.95 \mathrm{GeV} / c^{2}$. (b) Parameter $\lambda$ as a function of $x_{\pi}$. The curves are from high-twist predictions of Ref. 28, Figures taken from Ref. 21

\subsection{Theoretical Interpretations}

As described above, there are two phenomena in the angular distributions of pioninduced Drell-Yan processes which are beyond conventional perturbative QCD: longitudinally polarized virtual photons at large $x_{\pi}$ and violation of Lam-Tung relation at large $p_{T}$. Several attempts have been made in understanding their origins and some of them are introduced below.

\subsection{1. longitudinally polarized virtual photons at large $x_{\pi}$}

Berger and Brodsky ${ }^{28}$ explained the change of virtual photon polarization near $x_{\pi}=1$ by higher-twist QCD effect. The diagram of scattering of pions as two valence partons with an exchange of gluons between each other or the pion valance anti-quark and quark of target nucleon becomes dominant. The angular distribution was described as follows:

$$
\frac{d \sigma}{d \Omega} \propto\left(1-x_{\pi}\right)^{2}\left(1+\cos ^{2} \theta\right)+\frac{4 x_{\pi}^{2}\left\langle k_{T}^{2}\right\rangle}{9 m_{l \bar{l}}^{2}}\left(1-\cos ^{2} \theta\right)
$$

where $\left\langle k_{T}^{2}\right\rangle$ is the average of the square of transverse momentum of pion valance anti-quark.

In comparison with Eq. (1), $\lambda$ is 1 at small $x_{\pi}$ and gradually turns to be -1 as 

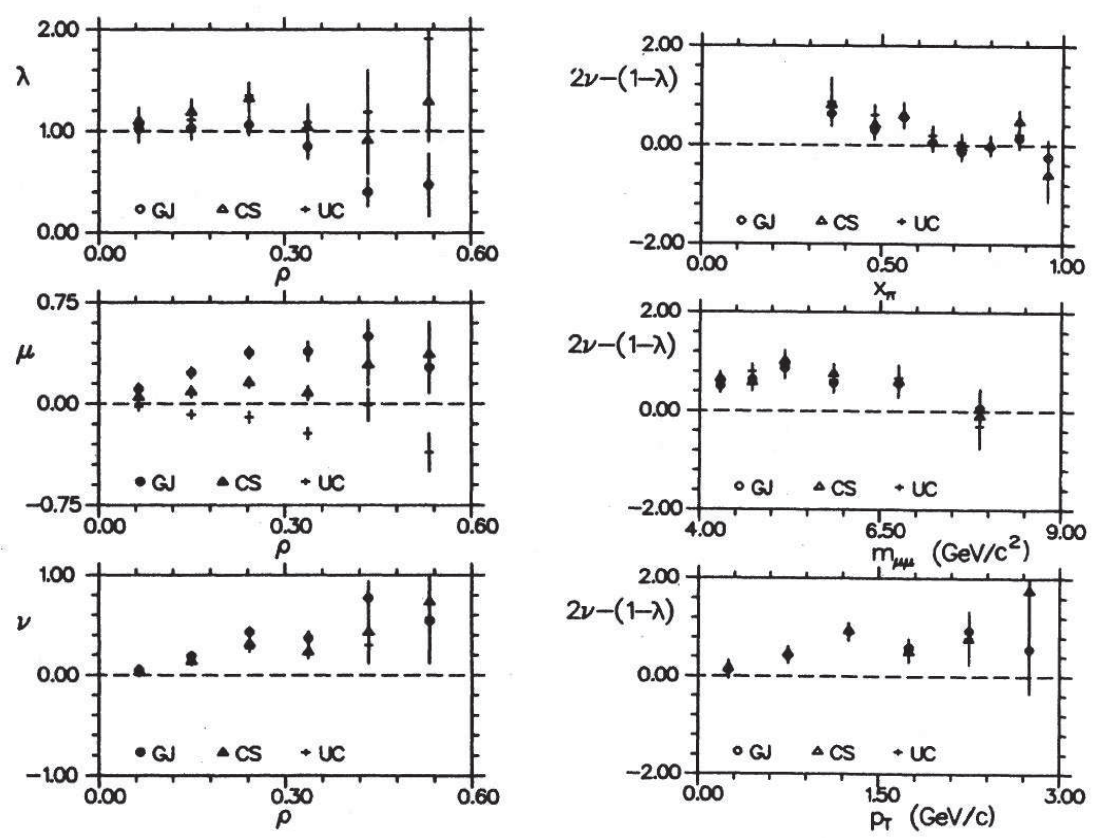

Fig. 2. (a) Angular parameters $\lambda, \mu$ and $\nu$ as a function of $\rho \equiv p_{T} / m_{l \bar{l}}$ in GJ, CS and u-channel frames. (b) Test of the Lam-Tung relation $1-\lambda=2 \mu$. Figures taken from Ref. 21

$x_{\pi} \rightarrow 1$. Furthermore it has been illustrated that the $x_{\pi}$-dependence of $\lambda, \mu$ and $\nu$ in the angular distributions of leptons are sensitive to the pion distribution amplitude (DA) ${ }^{29}$ which represents the distribution of light-cone momentum fraction in the Fock state of two valence quarks for pion. Pion DA is an important component for QCD light-cone sum rule and QCD factorization theory in describing the exclusive processes, e.g. $\gamma \gamma^{*} \rightarrow \pi^{0}$ the pion-photon transition form factor at $e^{+} e^{-}$collisions. ${ }^{30}$ Recent investigation 31 taking into account of QCD evolution of pion DA shows that the sensitivity of dilepton angular distributions to pion DA still remains, especially for the lepton pairs with large $\rho\left(\equiv p_{T} / m_{l \bar{l}}\right)$.

\subsubsection{Violation of Lam-Tung relation at large $p_{T}$}

A general two-particle spin-density matrix for the $q \bar{q}$ pair prior to the annihilation is described by $\frac{26}{322}$

$$
\rho^{(q, \bar{q})}=\frac{1}{4} \mathbb{1}^{q} \otimes \mathbb{1}^{\bar{q}}+F_{j} \sigma_{j}^{q} \otimes \mathbb{1}^{\bar{q}}+G_{j} \mathbb{1}^{q} \otimes \sigma_{j}^{\bar{q}}+H_{i j} \sigma_{i}^{q} \otimes \sigma_{j}^{\bar{q}} .
$$

The quantities $F_{j}, G_{j}$ and $H_{i j}$ characterizes different parts of spin configurations. In the scheme of non-polarized $q$ and $\bar{q}$, only the diagonal term survives $\rho^{(q, \bar{q})}=$ $\frac{1}{4} \mathbb{1}^{q} \otimes \mathbb{1}^{\bar{q}}$. 
The interference between opposite photon helicities gives rise to a $\nu$ or $\cos 2 \phi$ asymmetry and this means that the annihilated $q$ and $\bar{q}$ carry specific helicity configurations. The observation of violation of the Lam-Tung relation suggests certain mechanisms correlating the helicities of quark and anti-quark which comes from two individual hadrons. Such an effect is commonly expected to be of non-perturbative QCD in origin.

Brandenburg et al.$^{26}$ proposed a factorization breaking effect caused by nontrivial QCD vacuum. Through the spin-flip gluon synchrotron emission and Chromomagnetic Sokolov-Ternov effect, a spin correlation between an annihilating quark and anti-quark happens. ${ }^{33 \mid 34}$ Thus $H_{i j}$ becomes non-zero and in general there is no factorization of the spin density matrix. It was shown that a non-zero correlation coefficient $\kappa$ could describe the observed Lam-Tung violation:

$$
\kappa \equiv \frac{H_{22}-H_{11}}{1+H_{33}} \approx-\frac{1}{4}(1-\lambda-2 \nu) .
$$

The $q \bar{q}$ spin-density matrix becomes entangled and cannot be factorized. Since this effect originates from the property of QCD vacuum, it is expected to be independent of quark (anti-quark) flavor and the momentum fraction $x$ and and the amount of violation is predicted to persist at large $p_{T}$.

On the other hand, Boer ${ }^{35}$ considered a hadronic effect where the spin direction of quark (anti-quark) correlates with its transverse momentum $\left(k_{T}\right)$ within an unpolarized hadron itself. This correlation function "Boer-Mulders functions" $\left(h_{1}^{\perp}\left(x, k_{T}^{2}\right)\right)$ describes the unbalance of number densities of quarks with opposite transverse polarization with respect to the unpolarized hadron momentum. ${ }^{36}$ It is one of the key component of TMD parton distributions to be extracted from unpolarized hadrons. In this approach, the $q \bar{q}$ spin-density matrix becomes factorized as the production of two nontrivial one-particle spin-density matrices: $\rho^{(q, \bar{q})}=\frac{1}{4} \mathbb{1}^{q} \otimes \mathbb{1}^{\bar{q}}+F_{j} \sigma_{j}^{q} \otimes \mathbb{1}^{\bar{q}}+G_{j} \mathbb{1}^{q} \otimes \sigma_{j}^{\bar{q}}$ and $\kappa=\nu / 2 \propto h_{1}^{\perp}\left(q_{N}\right) h_{1}^{\perp}\left(\bar{q}_{\pi}\right)$. In general $h_{1}^{\perp}$ has the quark-flavor and $x$ dependence and is parametrized to vanish at large $k_{T}^{2}$.

Recently FNAL E866 experiment measured proton-induced Drell-Yan process with protons ${ }^{37}$ and deuterons ${ }^{38}$ but did not observe the violation of Lam-Tung relation as in the pion-induced one. This result could be interpreted as the smallness of Boer-Mulders function for sea quarks in the target nucleons but is less compatible with the supposed flavor-blind QCD vacuum effect. In addition no violation of LamTung relation was seen in the anti-proton-induced Drell-Yan process by CDF 39 Since this measurement was made in the region of large $m_{l \bar{l}}$ at Z-pole and very large $p_{T}$, the result might not be sensitive to the proposed non-perturbative QCD effect.

Besides the above two attempts, there are considerations of QCD instantoninduced effect ${ }^{40}$ and Glauber gluons in the $k_{T}$ factorization theorem ${ }^{41}$ As for the mechanism of Glauber gluons, a large Glauber phase solely for the pion is claimed and therefore it predicts that the violation of the Lam-Tung relation would be 
observed only with the pion beam but not with the other hadron beams. $\frac{41}{4}$ More precise measurement of dilepton angular distributions with pion beam over a wide kinematic range and with the other hadron beams like anti-proton and kaon, will help differentiate the theoretical interpretations.

\section{The EMC Effect}

In addition to probing the parton distribution functions in hadrons, the Drell-Yan process is also sensitive to modifications to the parton distributions for nucleons bound inside nuclei. However, since the energy scale probed in experiments studying the partonic structure is orders of magnitude larger than the nuclear binding energy, the nuclear parton distributions were expected to be the same as those of the nucleons.

In 1983, a muon-induced a Deep Inelastic Scattering (DIS) experiment by the European Muon Collaboration (EMC) ${ }^{42}$ discovered that the nucleon structure functions measured on iron and deuterium were different. They observed a depletion of the iron structure functions at large $x$, which was dubbed as the "EMC effect". The EMC effect has since been confirmed over a broad range of nuclear masses $(A)$ and momentum transfers $\left(Q^{2}\right)$, by several DIS experiments using electron, 43

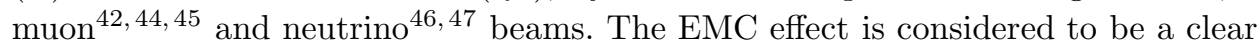
evidence that the quark distributions in nuclei are modified compared to those in the nucleons. As mentioned, because of the high energy scale probed in DIS experiments, the EMC effect was an unexpected observation. With the ever improving precision of the DIS experiments, specially the most recent experiments on light nuclei,$\sqrt[48]{49}$ they provide a detailed and multi-dimensional picture of the nuclear modification of the quark distributions. It is naturally expected that the changes in quark distributions inside nuclei should also manifest itself in other processes, where the quark distributions are important, such as the Drell-Yan process. ${ }^{3}[4$

The structure functions measured in DIS, determine only a combination of quark distributions rather the individual quark distributions. Thus it is possible, in principle, that models which give almost identical results for the EMC ratio can give different predictions for the Drell-Yan ${ }^{50 \mid 51}$ process. The EMC effect has indeed been found to be experimentally consistent (in the time-like region) with both the pionand proton-induced Drell-Yan processes 6 6/7|20|52] Since the sea quark distributions are less well known the results from proton-induced Drell-Yan results are far less conclusive in verifying the EMC effect.

Pion-induced Drell-Yan processes are relatively more sensitive to the EMC effect, specially the cross sections ratios $\frac{\sigma^{D Y}\left(\pi^{-}+A\right)}{\sigma^{D Y}\left(\pi^{-}+\mathrm{D}\right)}, \frac{\sigma^{D Y}\left(\pi^{-}+A\right)}{\sigma^{D Y}\left(\pi^{-}+\mathrm{H}\right)}$, and $\frac{\sigma^{D Y}\left(\pi^{+}+A\right)}{\sigma^{D Y}\left(\pi^{-}+A\right)}$, where $A$

represents a nuclear, $\mathrm{D}$ a deuteron and $\mathrm{H}$ a hydrogen target. Assuming isospin symmetry, which implies $u_{\pi^{+}}=d_{\pi^{-}}, \bar{u}_{\pi^{-}}=\bar{d}_{\pi^{+}}, \bar{u}_{\pi^{+}}=\bar{d}_{\pi^{-}}, u_{\pi^{-}}=d_{\pi^{+}}$and keeping only the dominant terms in each cross-section, we get 


$$
\begin{aligned}
& R_{A / D}^{-}=\frac{\sigma^{D Y}\left(\pi^{-}+A\right)}{\sigma^{D Y}\left(\pi^{-}+\mathrm{D}\right)} \approx \frac{u_{A}(x)}{u_{D}(x)}, \\
& R_{A / H}^{-}=\frac{\sigma^{D Y}\left(\pi^{-}+A\right)}{\sigma^{D Y}\left(\pi^{-}+\mathrm{H}\right)} \approx \frac{u_{A}(x)}{u_{p}(x)}, \\
& R_{ \pm}=\frac{\sigma^{D Y}\left(\pi^{+}+A\right)}{\sigma^{D Y}\left(\pi^{-}+A\right)} \approx \frac{d_{A}(x)}{4 u_{A}(x)} .
\end{aligned}
$$

The target quark distributions have a subscript $A$, and the up quark distribution in the deuteron and the proton are labeled by $u_{D}$ and $u_{p}$, respectively. It is clear that pion-induced Drell-Yan cross-section ratios, specially those between a heavy nuclear target and a hydrogen or deuterium target, should be sensitive to the EMC effect and, importantly, they are not sensitive to the pion structure functions, which are not yet accurately determined.
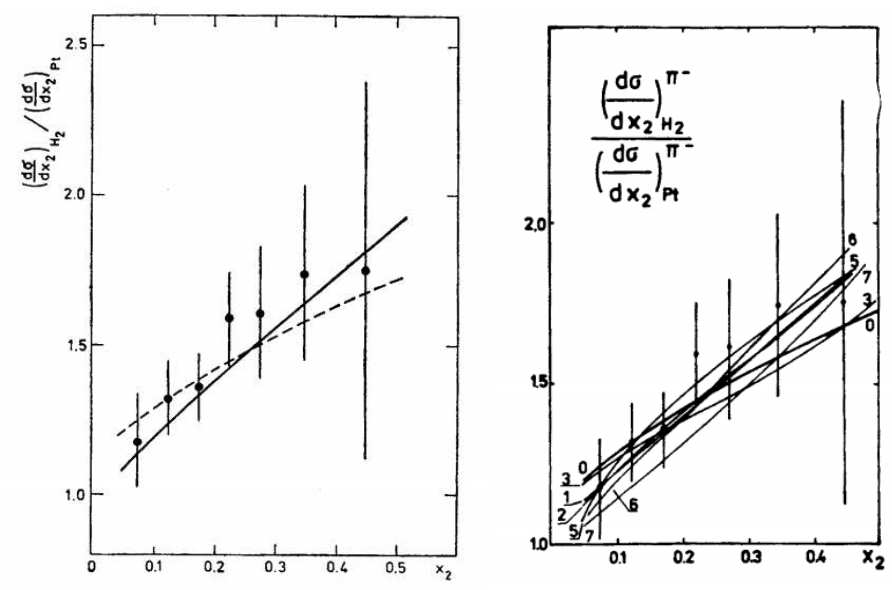

Fig. 3. (left)The ratio $\frac{\sigma^{D Y}\left(\pi^{-}+H\right)}{\sigma^{D Y}\left(\pi^{-}+\mathrm{Pt}\right)}$ vs $x_{2}$ the Bjorken scaling variable for the interacting quark of the nucleon in the target nucleus. The data are from the NA3 experiment and they are compared to a fit to the EMC data with (solid line) and without (dashed) corrections for $\Delta$ isobars. (right) The ratio $\frac{\sigma^{D Y}\left(\pi^{-}+H\right)}{\sigma^{D Y}\left(\pi^{-}+\mathrm{Pt}\right)}$ vs $x_{2}$ from the NA3 data compared to seven different models which reproduce the EMC effect. Figures taken from Ref. 51

The existing set of pion-induced Drell-Yan data that can be used to form these ratios are primarily from three experiments; the NA3, the NA10 and the Omega experiments. Chmaj and Heller ${ }^{50 \mid 51}$ have compared the ratio $\frac{\sigma^{D Y}\left(\pi^{-}+H\right)}{\sigma^{D Y}\left(\pi^{-}+\mathrm{Pt}\right)}$, measured by the NA3 collaboration, $\frac{16}{16}$ with fits to the EMC data as well as a wide variety of models that accurately reproduce the EMC effect. They found that indeed the DrellYan cross section ratios are sensitive to the EMC effect (see Fig. 3), however, most 
models available in 1985, which could reproduce the EMC data, gave predictions for the Drell-Yan process which have little or no difference. The results of their comparison of the NA3 data with a fit to the EMC data and seven widely different models, that all reproduced the EMC effect, is shown in Fig. 3, They concluded that the cross-section ratio obtained from the NA3 data confirm the modification of the quark distributions inside the nucleus (i.e. the EMC effect) however they are not precise enough to distinguish between the various models of the EMC effect.

The data from the NA10 experiment ${ }^{20 \mid}$ was used to form the ratio $\frac{\sigma^{D Y}\left(\pi^{-}+W\right)}{\sigma^{D Y}\left(\pi^{-}+D\right)}$ for the $286 \mathrm{GeV}$ and $140 \mathrm{GeV} \pi^{-}$beam, as well as both data sets combined together. In Fig. 4, the ratio for the combined data set is shown vs $x_{1}$ the Bjorken scaling variable for the interacting quark in the pion, $x_{2}$ the analogous quantity for the nucleon in the target nucleus, $\sqrt{\tau} \approx \sqrt{x_{1} x_{2}}$ and $x_{F} \approx x_{1}-x_{2}$. The data are compared to the ratio of structure functions obtained from the DIS experiment by the BCDMS collaboration $\underline{44}$ The NA10 Drell-Yan data were completely consistent with the modification of the nucleon quark distributions in the nucleus, while the pion quark distributions were found to be unaffected, all consistent with QCD factorization.

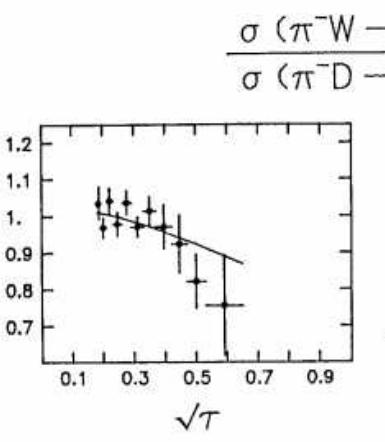

$\left.\rightarrow \mu^{+} \mu^{-}+X\right)$
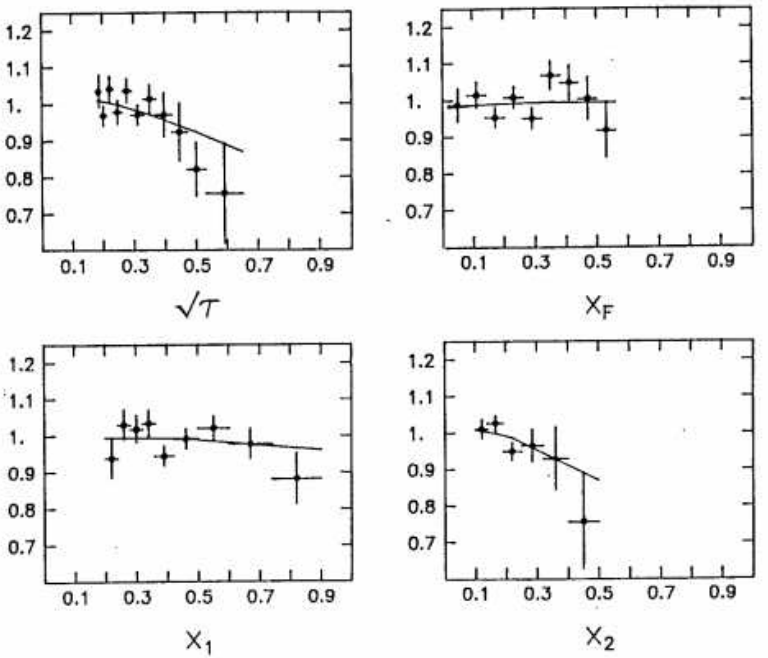

Fig. 4. The ratio $\frac{\sigma^{D Y}\left(\pi^{-}+W\right)}{\sigma^{D Y}\left(\pi^{-}+\mathrm{D}\right)}$ for the combined $286 \mathrm{GeV}$ and $140 \mathrm{GeV}$ data, $\frac{20}{20 m p a r e d}$ to the model predictions based on the BCDMS results 4445 


\section{Quark flavor-dependent EMC effect}

The specific origins of the observed $A$ dependence of the nuclear quark distributions have yet to be unambiguously identified. Attempts to explain the EMC effect have led to an extensive collection of theoretical models, $\frac{53[54}{54}$ that describe the essential features of the data, however the important physics of these models is often very different. This suggests that there are aspects of the EMC effect that are not probed in DIS, and new experimental observables are essential to understand the origins of the EMC effect. The quark flavor dependence of the EMC effect is one such promising experimental observable. In the simplest picture where the nuclear parton distribution functions (PDFs) are just the nucleon PDFs smeared by the Fermi motion of the nucleons, and thus the nuclear modification would be very similar for $u$ and $d$ quarks 55 In contrast, some models such as the pion-excess mode $\sqrt{56}+58$ predicts a flavor-dependent nuclear modifications arising from the different isospin composition of the pion cloud of protons and neutrons. Similarly, a recent model, the Cloët, Bentz and Thomas (CBT) reported in Refs. 559, 55, claims that for $N \neq Z$ nuclei (where $N$ and $Z$ refer to the number of neutrons and protons, respectively) the isovector-vector mean field (usually denoted by $\rho^{0}$ ) will affect the $u$ quarks differently from the $d$ quarks in the bound nucleons. Therefore, this model predicts that the $u$ and $d$ quarks have distinct nuclear modifications influenced by the $N / Z$ ratio of the nucleus, ${ }^{59}$ leading to a flavor sensitive EMC effect in nuclei with $N \neq Z$.

Pion-induced Drell-Yan processes are an experimental tool that is sensitive to flavor-dependent effects in the nuclear quark distributions. We have already noted that the cross section ratios of pionic Drell-Yan processes (Eqs. (5)-(7)) are sensitive to the EMC effect. Moreover, they are also sensitive to the flavor dependence of the EMC effect. The Drell-Yan ratio, $R_{ \pm}$, of Eq. (7) is an ideal experimental observable to search for flavor-dependent EMC effect, since it is directly proportional to $d_{A}(x) / u_{A}(x)$. The only existing data on $R_{ \pm}$are from the Omega collaboration. 17 In Ref. 60, the CBT model with and without the flavor dependence was compared to the existing pionic Drell-Yan data as shown in Fig. 5 (a-d). From the analysis of Ref. 60 it is clear that the flavor-dependent model is preferred for the NA3 and the Omega collaborations data sets, whereas the NA10 results are better described by the flavor-independent CBT model. Unfortunately, the existing data lack the precision needed to place a useful constraint on the flavor dependence of the EMC effect.

The COMPASS collaboration ${ }^{61}$ proposes to measure the Drell-Yan crosssections with $190-\mathrm{GeV} / c$ pion beams which will be an opportunity to test the flavordependence of the EMC effect. The sensitivity of the proposed COMPASS measurement was examined in Ref. 60 and is shown in Fig. $5(\mathrm{e}-\mathrm{h})$. The significant difference between the predicted ratios for the flavor-dependent versus flavor-independent nuclear PDFs provide a strong motivation for these future measurements. 

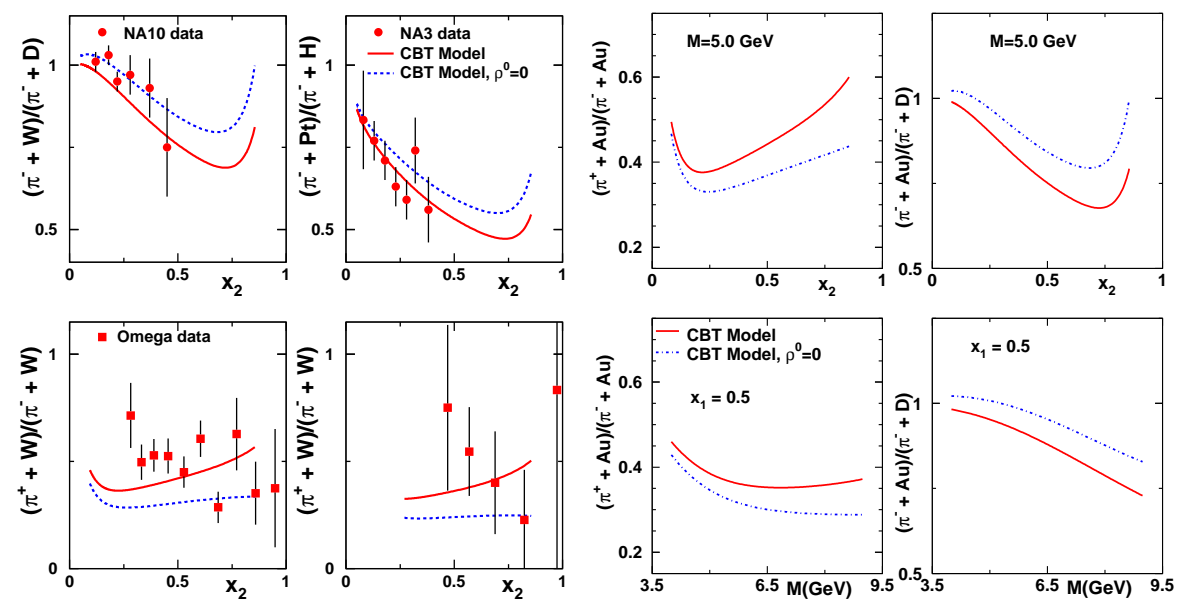

Fig. 5. The existing data for the ratios (a) $\frac{\sigma^{D Y}\left(\pi^{-}+\mathrm{W}\right)}{\sigma^{D Y}\left(\pi^{-}+\mathrm{D}\right)}$, (b) $\frac{\sigma^{D Y}\left(\pi^{-}+\mathrm{Pt}\right)}{\sigma^{D Y}\left(\pi^{-}+\mathrm{H}\right)}$ and $\frac{\sigma^{D Y}\left(\pi^{+}+\mathrm{W}\right)}{\sigma^{D Y}\left(\pi^{-}+\mathrm{W}\right)}$ for (c) $5.3 \leq Q^{2} \leq 7.2 \mathrm{GeV}^{2}$ and (d) $16 \leq Q^{2} \leq 25 \mathrm{GeV}^{2}$. The curves are results using the nuclear PDFs from the CBT model with $N / Z$ equal to that of tungsten. The solid line is the full flavor-dependent result and the dashed line is obtained by setting the $\rho^{0}$ mean-field to zero. The Drell-Yan crosssection ratios for (e) $\frac{\sigma^{D Y}\left(\pi^{+}+\mathrm{Au}\right)}{\sigma^{D Y}\left(\pi^{-}+\mathrm{Au}\right)}$ and (f) $\frac{\sigma^{D Y}\left(\pi^{-}+\mathrm{Au}\right)}{\sigma^{D Y}\left(\pi^{-}+\mathrm{D}\right)}$ as a function of $x_{2}$, using nuclear PDFs from the CBT model for gold (red solid) and the CBT model with the $\rho^{0}$ mean-field set to zero (blue dot-dashed). (g) and $(\mathrm{h})$ show the same ratios as a function of the dimuon mass, at a fixed $x_{1}=0.5$. Figures taken from Ref. 60

\section{Future Programs}

\subsection{Polarized pion-induced Drell-Yan in COMPASS-II at CERN}

As mentioned in Sec. 2.1.2 the angular distributions of leptons from Drell-Yan process may be sensitive to a key component of the TMD parton distributions known as the Boer-Mulders function. ${ }^{36}$ The other important single spin asymmetry (SSA) component in TMD parton distributions which could also be explored by the Drell-Yan process is the Sivers function $\left(f_{1 T}^{\perp}\right){ }^{62}$ This function describes how the transverse momentum $k_{T}$ distribution of unpolarized partons is distorted by the transverse polarization of the parent hadron. The correlation between $k_{T}$ and parton/hadron transverse polarization is intuitively resulted from a non-vanishing orbital angular momentum of the quarks themselves.

Sivers function, formerly considered to be zero by the time-reversal property of $\mathrm{QCD}, \sqrt[63]{ }$ was found to survive because of the presence of a gauge line (Wilson line) for the need of gauge invariance in the definition of TMD parton distribution. 64 This single spin asymmetry has been clearly observed in the semi-inclusive deeply inelastic scattering process (SIDIS) by HERMES experiment at DESY COMPASS experiment at CERN 68 That has allowed an extraction of Sivers function with global fits ${ }^{72}+75$ and opposite contributions from $u$ and $d$ quarks are seen. 
Sivers function could also be extracted from the polarized Drell-Yan process with the great advantage of it being free from convolution of fragmentation function. There exists an essential prediction that the $f_{1 T}^{\perp}$ functions extracted from DrellYan processes and those obtained from SIDIS must be reserved in sign because the Wilson line happens in the initial and final state respectively ${ }^{64}$ The same prediction also applies for the Boer-Mulders function to be determined in SIDIS and Drell-Yan processes. An experimental verification of the sign-reversal property of the Sivers and Boer-Mulders functions is a crucial test of QCD in the non-perturbative regime on the aspects of the origin of SSAs and the validity of factorization scheme - the corner stone of TMD physics.

A proposa $\sqrt{61}$ to carry out a polarized Drell-Yan measurement by COMPASS experiment in 2014 at CERN was approved. The angular distributions of Drell-Yan events produced by $190-\mathrm{GeV} / c \pi^{-}$beam colliding with transversely polarized $\mathrm{NH}_{3}$ target will be measured ${ }^{76} \mid 77$ Both the Sivers and Boer-Mulders functions from the target quark and the beam anti-quark could be extracted from the azimuthal spin asymmetries for testing the universality of the Sivers and Boer-Mulders functions between Drell-Yan and SIDIS. It should be noted that an unpolarized Drell-Yan program using liquid hydrogen, liquid deuterium and nuclei targets in COMPASS experiment will be very helpful to further investigate the known interesting phenomena in the dilepton angular distributions and the EMC effect.

\subsection{Exclusive pion-induced Drell-Yan at J-PARC}

When $x_{\pi}$ approaches the limit of 1 , the inclusive Drell-Yan process actually becomes an exclusive one: $\pi^{-} N \rightarrow N^{\prime} \mu^{+} \mu^{-}$. There are recent theoretical studies on the exclusive pion-induced Drell-Yan process where the nucleon remains intact in the final state $\frac{78}{86}$ This process is a time-like crossing of the deeply virtual pion production process. A factorization of this process shows that three important nonperturbative components are involved: nucleon GPD, pion DA and nucleon-to-pion transition distribution amplitude (TDA), as shown in Fig. 6.
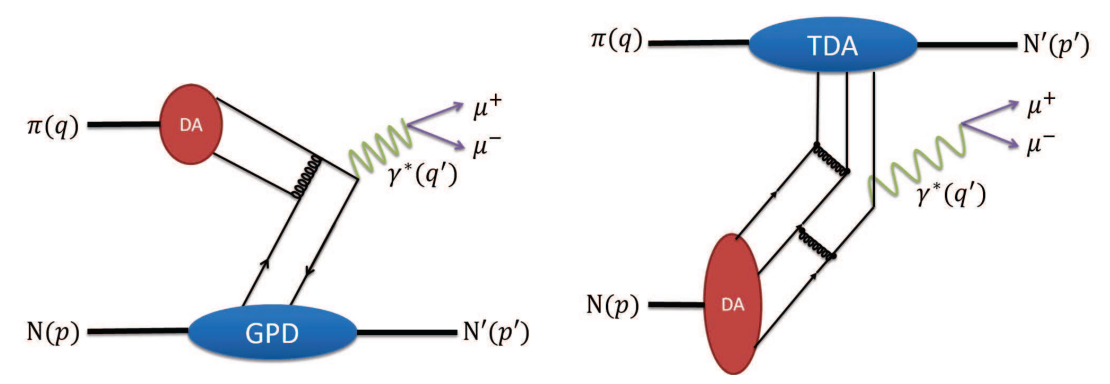

Fig. 6. Exclusive pion-induced $2 \rightarrow 2$ processes: with (a) small and (b) large momentum-transfer to the nucleon target 88 
The GPDs contain information of the correlation between the quark/gluon transverse position in the nucleon and its longitudinal momentum, which has been assessed by deeply virtual Compton scattering (DVCS) and deep virtual meson production (DVMP). The distribution amplitudes of nucleons probe the three-quark component of the nucleon light-cone wave function, while higher order components in the Fock-space expansion of the nucleon state are essential to describe the nucleon-to-pion transition distribution amplitudes (TDA) 87 When studying the nucleon structure, the nucleon-to-pion TDAs are particularly interesting because they directly probe the three-quark plus sea $q \bar{q}$ pair component, $\psi_{(3 q+q \bar{q})}$, which is related to the pion-cloud component inside the nucleon.

Since the cross sections of exclusive processes is larger at low CMS energies, it is appropriate to carry out such kind of measurement with pion beam of lower momentum. An approved three-year project of constructing a high-momentum beam line (HiPBL) in the Hadron Hall at J-PARC ${ }^{88}$ started from year 2013. Upon completion of HiPBL, high-flux primary proton beam and secondary particles, e.g. $\pi$, $K$ or $\bar{p}$ in the momentum range of $15-20 \mathrm{GeV} / c$ would be available for the study of hadron physics. An exclusive Drell-Yan process could be experimentally characterized if the scattered nucleons in the final state be either directly detected, or identified via the missing-mass technique. This measurement is very challenging considering the very small production cross section in the order of pico barn and a requirement of good momentum resolution for the decayed leptons passing through hadron absorber. Nevertheless the results in the time-like region, if available, will be complementary to those in space-like region assessed by the DVCS and DVMS processes to be studied in JLab. 89

\section{Conclusion}

We summarize the results of dilepton angular distributions and the nuclear modification of the parton distributions from the pion-induced Drell-Yan process. The discrepancies between the observed dilepton angular distributions and the expectations of the Drell-Yan parton model together with perturbative QCD corrections, can be resolved by incorporating the non-perturbative QCD physics associated with the bound-state effects of the partons. These connections open up a clean and important way of accessing the TMD distribution and GPD of nucleons, and pion DA. Also the study of pion-induced Drell-Yan process on heavy nuclei will help reveal the very interesting flavor-dependent modification of quark distributions in the nuclear medium. With several upcoming experiments and others being planned, we expect that pion-induced Drell-Yan process should continue improving our understanding of QCD and the hadron structure in multi-dimensions.

\section{Acknowledgments}

This work is supported in part by the U.S. Department of Energy (Grant No. DE-FG02-07ER41528) and the National Science Council of Taiwan (Grant No. 
100-2112-M-001-015-MY3).

\section{References}

1. J. Christenson et al., Phys. Rev. Lett. 25, 1523 (1970).

2. J. Christenson et al., Phys. Rev. D8, 2016 (1973);

3. S.D. Drell and T.M. Yan Phys. Rev. Lett. 25, 316 (1970).

4. S.D. Drell and T.M. Yan Phys. Rev. Lett. 25, 902 (1970).

5. I.R. Kenyon, Rep. Prog. Phys. 45, 1261 (1982).

6. C. Grosso-Pilcher and M.J. Shochet, Annu. Rev. Nucl. Part. Sci. 36, 1 (1986).

7. P.L. McGaughey, J.M. Moss, and J.C. Peng, Annu. Rev. Nucl. Part. Sci. 49, 217 (1999).

8. E. Perez and E. Rizvi, Rep. Prog. Phys. 76, 046201 (2013) and references therein.

9. V. Barone, F. Bradamante, A. Martin, Prog. Part. Nucl. Phys. 65, 267 (2010) and references therein.

10. Durham database of pionic Drell-Yan production, http://durpdg.dur.ac.uk/spires/hepdata/online/dy/pi-Nto2muX.html and http://durpdg.dur.ac.uk/spires/hepdata/online/dy/pi+Nto2muX.html

11. C.B. Newman et al. (CIP Collaboration), Phys. Rev. Lett. 42,948 (1979).

12. K.J. Anderson et al. (CIP Collaboration), Phys. Rev. Lett. 43,1219 (1979).

13. S. Palestini et al. (CIP Collaboration), Phys. Rev. Lett. 55,2649 (1985).

14. J.G. Heinrich et al. (CIP Collaboration), Phys. Rev. D44,1909 (1991).

15. J. Badier et al. (NA3 Collaboration), Z. Phys. C 11, 195 (1981).

16. A. Michelini, CERN-EP/81-128 (1981); J. Badier et al. (NA3 Collaboration), Phys. Lett. B 104, 335 (1981).

17. M. Corden et al. (Omega Collaboration), Phys. Lett. B 96, 417 (1980).

18. S. Falciano et al. (NA10 Collaboration), Z. Phys. C 31, 513 (1986).

19. M. Guanziroli et al. (NA10 Collaboration), Z. Phys. C 37, 545 (1988).

20. P. Bordalo et al. (NA10 Collaboration), Phys. Lett. B 193, 368 (1987).

21. J.S. Conway et al. (E615 Collaboration), Phys. Rev. D 39, 92 (1989).

22. M. Diehl, Phys. Rept. 388, 41 (2003) and references therein.

23. C.S. Lam and W.K. Tung, Phys. Rev. D 18, 2447 (1978).

24. C.S. Lam and W.K. Tung, Phys. Lett. B 80, 228 (1979).

25. C.S. Lam and W.K. Tung, Phys. Rev. D 21, 2712 (1980).

26. A. Brandenburg, O. Nachtmann, and E. Mirkes, Z. Phys. C 60, 697 (1993).

27. E. Mirkes and J. Ohnemus, Phys. Rev. D 51, 4891(1995)

28. E.L. Berger and S.J. Brodsky, Phys. Rev. Lett. 42, 940 (1979).

29. A. Brandenburg, S.J. Brodsky, V.V. Khoze, and D. Müller, Phys. Rev. Lett 73, 939 (1994).

30. X.-G. Wu, T. Huang, and T. Zhong, Chin. Phys. C 37, 063105 (2013)

31. A.P. Bakulev N.G. Stefanis, and O.V. Teryaev, Phys. Rev. D 76, 074032 (2007)

32. D. Boer, A. Brandenburg, O. Nachtmann, and A. Utermann, Eur. Phys. J. C40, 55 (2005).

33. O. Nachtmann and A. Reiter, Z. Phys. C 24, 283 (1984).

34. G.W. Botz, P. Haberl, and O. Nachtmann, Z. Phys. C 67, 143 (1995).

35. D. Boer, Phys. Rev. D 60, 014012 (1999).

36. D. Boer and P.J. Mulders, Phys. Rev. D 57, 5780 (1998).

37. L.Y. Zhu et al. (FNAL E866/NuSea Collaboration), Phys. Rev. Lett. 99, 082301 (2007).

38. L.Y. Zhu et al. (FNAL E866/NuSea Collaboration), Phys. Rev. Lett. 102, 182001 (2009). 
39. T. Aaltonen et al. (CDF Collaboration), Phys. Rev. Lett. 106, 241801 (2011)

40. A. Brandenburg, A. Ringwald, and A. Utermann, Nucl. Phys. B 754, 107 (2005).

41. C.P. Chang and H.N. Li, arXiv:1305.4694

42. J.J. Aubert et al. (European Muon Collaboration), Phys. Lett. B 123, 275 (1983).

43. J. Gomez et al., Phys. Rev. D 49, 4348 (1994).

44. G. Bari et al., Phys. Lett. B 163, 282 (1985).

45. A.C. Benvenuti et al., Phys. Lett. B 189, 483 (1987).

46. A.M. Cooper et al., Phys. Lett. B 141, 133 (1984).

47. H. Abramowicz et al., Z. Phys. C 25, 29 (1984).

48. K. Ackerstaff et al. (HERMES Collaboration), Phys. Lett. B 475, 386 (2000).

49. J. Seely et al., Phys. Rev. Lett. 103, 202301 (2009).

50. T. Chmaj and K.J. Heller, Acta Phy. Polo. B15, 473 (1984).

51. T. Chmaj and K.J. Heller, Acta Phy. Polo. B16, 423 (1985).

52. D.M. Alde et al. (E772 Collaboration), Phys. Rev. Lett. 64, 2479 (1990).

53. D.F. Geesaman, K. Saito and A.W. Thomas, Ann. Rev. Nucl. Part. Sci. 45, 337 (1995).

54. P.R. Norton, Rept. Prog. Phys. 66, 1253 (2003).

55. I.C. Cloët, W. Bentz and A.W. Thomas, Phys. Rev. Lett. 102, 252301 (2009).

56. C.H. Llewellyn-Smith, Phys. Lett. B 128, 107 (1983).

57. M. Ericson and A.W. Thomas, Phys. Lett. B 128, 112 (1983).

58. E.L. Berger, F. Coester, and R.B. Wiringa, Phys. Rev. D 29, 398 (1984).

59. I.C. Cloet, W. Bentz, A.W. Thomas, Phys. Lett. B642, 210-217 (2006).

60. D. Dutta, J.-C. Peng, I.C. Cloet and D. Gaskell, Phys. Rev. C 83, 042201R (2011).

61. COMPASS Collaboration, CERN Report No. CERN/SP SLC 96-14, SPSC/P 297; The COMPASS-II Proposal, http://wwwcompass.cern.ch/compass/proposal/compass-II_proposal/compass-II_proposal.pdf 62. D. Sivers, Phys. Rev. D 41, 83 (1990); D. Sivers, Phys. Rev. D 43, 261 (1991).

63. J.C. Collins, Nucl. Phys. B 396, 161 (1993).

64. J.C. Collins, Phys. Lett. B 536, 43 (2002).

65. A. Airapetian et al. (HERMES Collaboration), Phys. Rev. Lett. 94, 012002 (2005).

66. L.L. Pappalardo et al. (HERMES Collaboration), Eur. Phys. J. A 38, 145 (2008).

67. A. Airapetian et al. (HERMES Collaboration), Phys. Rev. Lett. 103, 152002 (2009).

68. V.Y. Alexakhin, et al. (COMPASS Collaboration), Phys. Rev. Lett. 94, 202002 (2005).

69. M. Alekseev et al. (COMPASS Collaboration), Phys. Lett. B 673, 127 (2009).

70. M.G. Alekseev et al. (COMPASS Collaboration), Phys. Lett. B 692, 240 (2010).

71. C. Adolph et al. (COMPASS Collaboration), Phys. Lett. B 717, 383 (2012).

72. W. Vogelsang and F. Yuan, Phys. Rev. D 72, 054028 (2005).

73. J.C. Collins et al., Phys. Rev. D 73, 014021 (2006).

74. M. Anselmino et al., Eur. Phys. J. A 39, 89 (2009).

75. M. Anselmino et al., Phys. Rev. D 86, 014028 (2012).

76. O. Denisov (COMPASS Collaboration), Mod. Phys. Lett. A 24, 3033 (2009).

77. C. Quintans (COMPASS Collaboration), J. Phys. Conf. Ser. 295, 012163 (2011).

78. E.R. Berger, M. Diehl, and B. Pire, Phys. Lett. B 523, 265 (2001).

79. D. Muller, B. Pire, L. Szymanowski, and J. Wagner, Phys. Revs. D 86, 031502 (2012).

80. B. Pire and L. Szymanowski, Phys. Lett. B 622 (2005) 83.

81. B. Pire and L. Szymanowski, Phys. Lett. B 622, 83 (2005).

82. J.P. Lansberg, B. Pire, and L. Szymanowski, Phys. Rev. D 76, 111502(R) (2007).

83. J.P. Lansberg, B. Pire, and L. Szymanowski, Phys. Rev. D 75, 074004 (2007).

84. J.P. Lansberg, B. Pire, and L. Szymanowski, Phys. Rev. 77, 019902(E) (2008). 
85. J.P. Lansberg, B. Pire, and L. Szymanowski, AIP Conf. Proc. 892, 278 (2007).

86. J.P. Lansberg, B. Pire, and L. Szymanowski, arXiv:0709.2567

87. B. Pasquini, M. Pincetti, and S. Boffi, Phys. Rev. D 80, 014017 (2009).

88. S. Sawada, talk of "Hadron experimental hall and high-momentum beamline at JPARC" in KEK theory center workshop on Hadron physics with high-momentum hadron beams at J-PARC in 2013.

89. J. Dudek et al., Eur. Phys. J. A 48, 187 (2012). 